\title{
Late-Time Tails of Wave Propagation in Higher Dimensional Spacetimes
}

\author{
Vitor Cardoso, ${ }^{*}$ Shijun Yoshida ${ }^{\dagger}$ and Óscar J. C. Dias ${ }^{\ddagger}$ \\ Centro Multidisciplinar de Astrofísica - CENTRA, \\ Departamento de Física, Instituto Superior Técnico, \\ Av. Rovisco Pais 1, 1049-001 Lisboa, Portugal, \\ José P. S. Lemos ${ }^{\S}$ \\ Department of Physics, Columbia University, New York, NY 10027, \\ USA, \& Centro Multidisciplinar de Astrofísica - CENTRA, \\ Departamento de Física, Instituto Superior Técnico, \\ Av. Rovisco Pais 1, 1049-001 Lisboa, Portugal.
}

(Dated: September 4, 2018)

\begin{abstract}
We study the late-time tails appearing in the propagation of massless fields (scalar, electromagnetic and gravitational) in the vicinities of a $D$-dimensional Schwarzschild black hole. We find that at late times the fields always exhibit a power-law falloff, but the power-law is highly sensitive to the dimensionality of the spacetime. Accordingly, for odd $D>3$ we find that the field behaves as $t^{-(2 l+D-2)}$ at late times, where $l$ is the angular index determining the angular dependence of the field. This behavior is entirely due to $D$ being odd, it does not depend on the presence of a black hole in the spacetime. Indeed this tail is already present in the flat space Green's function. On the other hand, for even $D>4$ the field decays as $t^{-(2 l+3 D-8)}$, and this time there is no contribution from the flat background. This power-law is entirely due to the presence of the black hole. The $D=4$ case is special and exhibits, as is well known, the $t^{-(2 l+3)}$ behavior. In the extra dimensional scenario for our Universe, our results are strictly correct if the extra dimensions are infinite, but also give a good description of the late time behaviour of any field if the large extra dimensions are large enough.
\end{abstract}

PACS numbers: 04.70.-s, 04.30.Nk, 04.70.Bw, 11.25.-w

\section{INTRODUCTION}

It is an everyday life experience that light rays and waves in general travel along a null cone. For example, if one lights a candle or a lighter for five seconds and then turns it off, any observer (at rest relative to the object) will see the light for exactly five seconds and then suddenly fade out completely. Mathematically this is due to the well known fact that the flat space 4-dimensional Green's function has a delta function character and therefore has support only on the light cone. There are however situations where this only-on-the light cone propagation is lost. For instance, in a curved spacetime a propagating wave leaves a "tail" behind, as shown by DeWitt and Brehme's seminal work [1]. This means that a pulse of gravitational waves (or any massless field for that matter) travels not only along the light cone but also spreads out behind it, and slowly dies off in tails. Put it another way, even after the candle is turned off in a curved spacetime, one will still see its shinning light, slowly fading away, but never completely. This is due to

\footnotetext{
*Electronic address: vcardoso@fisica.ist.utl.pt

${ }^{\dagger}$ Electronic address: yoshida@fisica.ist.utl.pt

${ }^{\ddagger}$ Electronic address: oscar@fisica.ist.utl.pt

$\S$ Electronic address: lemos@physics.columbia.edu
}

backscattering off the potential [2] at very large spatial distances.

The existence of late-time tails in black hole spacetimes is by now well established, both analytically and numerically, in linearized perturbations and even in a non-linear evolution, for massless or massive fields [2-9]. This is a problem of more than academic interest: one knows that a black hole radiates away everything that it can, by the so called no hair theorem (see [10] for a nice review), but how does this hair loss proceed dynamically? A more or less complete picture is now available. The study of a fairly general class of initial data evolution shows that the signal can roughly be divided in three parts: (i) the first part is the prompt response, at very early times, and the form depends strongly on the initial conditions. This is the most intuitive phase, being the obvious counterpart of the light cone propagation. (ii) at intermediate times the signal is dominated by an exponentially decaying ringing phase, and its form depends entirely on the black hole characteristics, through its associated quasinormal modes $[11,12]$. (iii) a late-time tail, usually a power law falloff of the field. This power law seems to be highly independent of the initial data, and seems to persist even if there is no black hole horizon. In fact it depends only on the asymptotic far region. Mathematically each of these stages has been associated as arising from different contributions to the Green's function. The late-time tail is due to a branch cut [5]. The study of 
linearized (we note that non-linear numerical evolution also displays these tails, but here we shall work at the linearized level) perturbations in the black hole exterior can usually be reduced to the simple equation

$$
\left[\partial_{t}^{2}-\partial_{x}^{2}+V(x)\right] \Psi=0,
$$

where the potential $V(x)$ depends on what kind of field one is considering and also, of course, on the spacetime. A detailed study of the branch cut contribution by Ching, Leung, Suen and Young [6, 7] has provided analytical results for some specific but quite broad class of potentials. These analytical results concerning the late time tails were confirmed numerically.

It is not generally appreciated that there is another case in which wave propagation develops tails: wave propagation in odd dimensional flat spacetimes. In fact, the Green's function in a $D$-dimensional spacetime [1315] have a completely different structure depending on whether $D$ is even or odd. For even $D$ it still has support only on the light cone, but for odd $D$ the support of the Green's function extends to the interior of the light cone, and leads to the appearance of tails. It is hard to find good literature on this subject, but a complete and pedagogical discussion of tails in flat $D$-dimensional backgrounds can be found in [15].

A study of wave physics in higher dimensions is now, more than ever, needed. It seems impossible to formulate in four dimensions a consistent theory which unifies gravity with the other forces in nature. Thus, most efforts in this direction have considered a higher dimensional arena for our universe, one example being string theories which have recently made some remarkable achievements. Moreover, recent investigations [16] propose the existence of extra dimensions in our Universe in order to solve the hierarchy problem, i.e., the huge difference between the electroweak and the Planck scale, $m_{\mathrm{EW}} / M_{\mathrm{Pl}} \sim 10^{-17}$. The fields of standard model would inhabit a 4 -dimensional sub-manifold, the brane, whereas the gravitational degrees of freedom would propagate throughout all dimensions.

A first step towards the understanding of gravitational wave physics in higher dimensions was given by Cardoso, Dias and Lemos [14], by studying wave generation and propagation in generic $D$-dimensional flat spacetimes. Here we shall take a step further, by studying wave tails in higher dimensional black hole spacetimes. We will restrict the analysis to higher dimensional Schwarzschild black holes. As expected, if one now considers tails in higher dimensional black hole spacetimes two aspects should emerge: in odd dimensional spacetimes one expects the black hole contribution to the tail to be smaller than that of the background itself. Therefore for odd dimensions the tail should basically be due to the flat space Green's function. However, for even $D$-dimensional black hole spacetimes there is no background contribution, and one expects to see only the black hole contribution to the tail. A recent study by Barvinsky and Solodukhin [17] has showed that such tails may not be impossible to de- tect. Unfortunately, the weakness of gravitational waves impinging on Earth, make this an unlikely event. We note however that they worked with small length, compact extra-dimensions, whereas we shall consider large extra dimensions. Our results will be strictly correct if the extra dimensions are infinite, but also allow us to determine the correct answer if the large extra dimensions are large enough that the timescale for wave reflection at the boundaries is larger than the timescales at which the tail begins to dominate.

The evolution problem in a $D$-dimensional Schwarzschild background can be cast in the form (1), and we will show also that the potential can be worked out in such a way as to belong to the class of potentials studied in $[6,7]$. Therefore, their analytical results carry over to the $D$-dimensional Schwarzschild black holes as well. We will verify this by a direct numerical evolution. The main results are: the late-time behavior is dominated by a tail, and this is a power-law falloff. For odd dimensions the power-law is determined not by the presence of the black hole, but by the fact that the spacetime is odd dimensional. In this case the field decays as $\Psi \sim t^{-(2 l+D-2)}$, where $l$ is the angular index determining the angular dependence of the field. This is one of the most interesting results obtained here. One can show directly from the flat space Green's function that such a power-law is indeed expected in flat odd dimensional spacetimes, and it is amusing to note that the same conclusion can be reached directly from the analysis of $[6,7]$. For even dimensional spacetimes we find also a power-law decay at late times, but with a much more rapid decay, $\Psi \sim t^{-(2 l+3 D-8)}$. For even $D$, this power-law tail is entirely due to the black hole, as opposed to the situation in odd $D$. These results are strictly valid for $D>4$. Four dimensional Schwarzschild geometry is special, having the well known power-law tail $\Psi \sim t^{-(2 l+3)}$.

\section{A BRIEF SUMMARY OF PREVIOUS ANALYTICAL RESULTS FOR A SPECIFIC CLASS OF POTENTIALS}

In a very complete analysis, Ching, Leung, Suen and Young $[6,7]$ have studied the late-time tails appearing when one deals with evolution equations of the form (1), and the potential $V$ is of the form

$$
V(x) \sim \frac{\nu(\nu+1)}{x^{2}}+\frac{c_{1} \log x+c_{2}}{x^{\alpha}}, x \rightarrow \infty .
$$

By a careful study of the branch cut contribution to the associated Green's function they concluded that in general the late-time behavior is dictated by a power-law or by a power-law times a logarithm, and the exponents of the power-law depend on the leading term at very large spatial distances. The case of interest for us here, as we shall verify in the following section, is when $c_{1}=0$. Their conclusions, which we will therefore restrict to the $c_{1}=0$ 
case, are (see Table 1 in [6] or [7]):

(i) if $\nu$ is an integer the term $\frac{\nu(\nu+1)}{x^{2}}$ does not contribute to the late-time tail. We note this term represents just the pure centrifugal barrier, characteristic of flat space, so one can expect that indeed it does not contribute, at least in four-dimensional spacetime. We also note that since even dimensional spacetimes have on-light cone propagation, one may expect to reduce the evolution equation to a form containing the term $\frac{\nu(\nu+1)}{x^{2}}$ with $\nu$ an integer. We shall find this is indeed the case. Therefore, for integer $\nu$, it is the $\frac{c_{2}}{x^{\alpha}}$ term that contributes to the late-time tail. In this case, the authors of $[6,7]$ find that the tail is given by a power-law,

$$
\Psi \sim t^{-\mu}, \mu>2 \nu+\alpha, \alpha \text { odd integer }<2 \nu+3 .
$$

For this case ( $\alpha$ an odd integer smaller than $2 \nu+3$ ) the exponent $\mu$ was not determined analytically. However, they argue both analytically and numerically, that $\mu=$ $2 \nu+2 \alpha-2$. For all other real $\alpha$, the tail is

$$
\Psi \sim t^{-(2 \nu+\alpha)} \text {, all other real } \alpha .
$$

(ii) if $\nu$ is not an integer, then the main contribution to the late-time tail comes from the $\frac{\nu(\nu+1)}{x^{2}}$ term. In this case the tail is

$$
\Psi \sim t^{-(2 \nu+2)}, \text { non - integer } \nu .
$$

We will now see that for a $D$-dimensional Schwarzschild geometry the potential entering the evolution equations is asymptotically of the form (2) and therefore the results (3)-(5) can be used.

\section{THE EVOLUTION EQUATIONS AND LATE-TIME TAILS IN THE $D$-DIMENSIONAL SCHWARZSCHILD GEOMETRY}

Here, we shall consider the equations describing the evolution of scalar, electromagnetic and gravitational weak fields outside the $D$-dimensional Schwarzschild geometry. We shall then, based on the results presented in the previous section, derive the late-time tails form of the waves. We will find they are always a power-law falloff.

\section{A. The evolution equations and the reduction of the potential to the standard form}

The metric of the $D$-dimensional Schwarzschild black hole in $\left(t, r, \theta_{1}, \theta_{2}, . ., \theta_{D-2}\right)$ coordinates is [18]

$$
d s^{2}=-f d t^{2}+f^{-1} d r^{2}+r^{2} d \Omega_{D-2}^{2},
$$

with

$$
f=1-\frac{M}{r^{D-3}}
$$

The mass of the black hole is given by $\frac{(D-2) \Omega_{D-2} M}{16 \pi \mathcal{G}}$, where $\Omega_{D-2}=\frac{2 \pi^{(D-1) / 2}}{\Gamma[(D-1) / 2]}$ is the area of a unit $(D-2)$ sphere, and $d \Omega_{D-2}^{2}$ is the line element on the unit sphere $S^{D-2}$. We will only consider the linearized approximation, which means that we are considering wave fields outside this geometry that are so weak they do not alter this background. Technically this means that all covariant derivatives are taken with respect to the background metric (6). The evolution equation for a massless scalar field follows directly from the (relativistic) Klein-Gordon equation. After a separation of the angular variables with Gegenbauer functions (see [19] for details) we get that the scalar field follows (1) with a potential

$V_{\mathrm{s}}\left(r_{*}\right)=f(r)\left[\frac{a}{r^{2}}+\frac{(D-2)(D-4) f(r)}{4 r^{2}}+\frac{(D-2) f^{\prime}(r)}{2 r}\right]$,

where $r$ is a function of the tortoise coordinate $r_{*}$ according to $\frac{\partial r}{\partial r_{*}}=f(r)$. The constant $a=l(l+D-3)$ is the eigenvalue of the Laplacian on the hypersphere $S^{D-2}$, and $f^{\prime}(r)=\frac{d f(r)}{d r}$. $\quad l$ can take any nonnegative integer value. Of course the evolution equation is (1) where the variable $x$ is in this case the tortoise coordinate $r_{*}$. This is the standard form in which the potential is presented. However, one can collect the different powers of $r$ and get

$$
V_{\mathrm{s}}\left(r_{*}\right)=f(r)\left[\frac{\nu(\nu+1)}{r^{2}}+\frac{1}{r^{D-1}} \frac{(D-2)^{2} M}{4}\right],
$$

where

$$
\nu=l-2+\frac{D}{2} .
$$

Asymptotically for large $r_{*}$ one can show that

$$
\left.V_{\mathrm{s}}\left(r_{*}\right)\right|_{r_{*} \rightarrow \infty}=\frac{\nu(\nu+1)}{r_{*}^{2}}+\frac{1}{r_{*}^{D-1}} \frac{(D-2) M l}{D-4}(3-l-D) .
$$

This is strictly valid for $D>4$. In the $D=4$ case there is a logarithm term [2]. Notice that the coefficient $\nu$ appearing in the centrifugal barrier term $\frac{\nu(\nu+1)}{r_{*}^{2}}$ is, as promised, an integer for even $D$, and a half-integer for odd $D$. The gravitational evolution equations have recently been derived by Kodama and Ishibashi [20]. There are three kinds of gravitational perturbations, according to Kodama and Ishibashi's terminology: the scalar gravitational, the vector gravitational and the tensor gravitational perturbations. The first two already have their counterparts in $D=4$, which were first derived by Regge and Wheeler [21] and by Zerilli [22]. The tensor type is a new kind appearing in higher dimensions. However, it obeys exactly the same equation as massless scalar fields, so the previous result (9)-(11) holds. It can be shown in fact that the scalar and vector type also obey the same evolution equation with a potential that also has the form (11) with a slightly different coefficient for the $1 / r_{*}^{D-1}$ term. For example, for the vector type the potential is

$$
V_{\mathrm{gv}}\left(r_{*}\right)=f(r)\left[\frac{\nu(\nu+1)}{r^{2}}-\frac{1}{r^{D-1}} \frac{3(D-2)^{2} M}{4}\right],
$$


where $\nu$ is defined in (10). Therefore asymptotically for large $r_{*}$,

$$
\begin{aligned}
\left.V_{\mathrm{gv}}\left(r_{*}\right)\right|_{r_{*} \rightarrow \infty}= & \frac{\nu(\nu+1)}{r_{*}^{2}}-\frac{1}{r_{*}^{D-1}} \frac{(D-2) M}{D-4} \times \\
& {\left[8+D^{2}+D(l-6)+l(l-3)\right] . }
\end{aligned}
$$

which is of the same form as the scalar field potential. The scalar gravitational potential has a more complex form, but one can show that asymptotically it has again the form (11) or (13) with a different coefficient in the $1 / r_{*}^{D-1}$ term. Since the explicit form of this coefficient is not important here, we shall not give it explicitly. Electromagnetic perturbations in higher dimensions were considered in [23]. Again, asymptotically for large $r_{*}$ they can be reduced to the form (11)(where again $\nu=l-2+\frac{D}{2}$ and the $1 / r_{*}^{D-1}$ coefficient is different), so we shall not dwell on them explicitly.

\section{B. Late-time tails}

Now that we have shown that the potentials appearing in evolution of massless fields in the $D$-dimensional Schwarzschild geometry belong to the class of potentials studied in $[6,7]$ we can easily find the form of the latetime tail. For odd dimensional spacetimes, $\nu$ is not an integer, therefore the centrifugal barrier gives the most important contribution to the late-time tail. According to (5) we have that the late-time tail is described by the power-law falloff

$$
\Psi \sim t^{-(2 l+D-2)} \text {, odd } D .
$$

According to the discussion in the introduction, this tail is independent of the presence of the black hole, and should therefore already appear in the flat space Green's function. Indeed it does $[14,15]$. The flat, odd dimensional Green's function has a tail term $[14,15]$ proportional to $\frac{\Theta(t-r)}{\left(t^{2}-r^{2}\right)^{D / 2-1}}$, where $\Theta$ is the Heaviside step function. It is therefore immediate to conclude that, for spherical perturbations, for example, the tail at very large times should be $t^{-(D-2)}$ which is in agreement with (14) for $l=0$ (which are the spherical perturbations). It is amusing to note that the analysis of $[6,7]$ gives the correct behavior at once, simply by looking at the centrifugal barrier! We have checked numerically the result (14) for $D=5$, and the results are shown in Figs. 1 and 2.

The numerical procedure followed the one outlined in [3], with constant data on $v=v_{0}$, where $v$ is the advanced time coordinate, $v=t+r_{*}$. One final remark is in order here. When numerically evolving the fields using the scheme in [3], we have found that for $D>5$ the tail looked always like $t^{-(2 \nu+4)}$. This is a fake behavior, and as pointed out in [7] (see in particular their Appendix A) it is entirely due to the ghost potential, appearing for potentials vanishing faster than $\frac{1}{r_{*}^{4}}$ for a

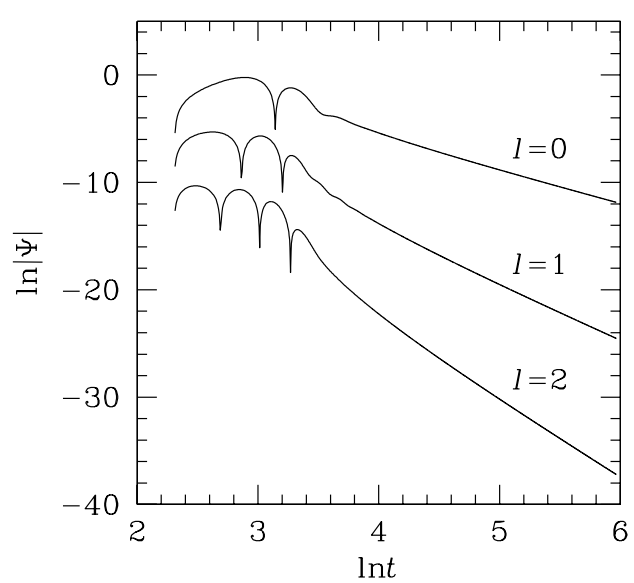

FIG. 1: Generic time dependence of a scalar field $\Psi$ in a five dimensional Schwarzschild geometry, at a fixed spatial position. We took as initial conditions a Gaussian wave packet with $\sigma=3$ and $v_{c}=10$. We have performed other numerical extractions for different initial values. The results for the late-time behavior are independent of the initial data, as far as we can tell. For $l=0$ the late-time behavior is a powerlaw with $\Psi \sim t^{-3.1}$, for $l=1, \Psi \sim t^{-5.2}$ at late times and for $l=2, \Psi \sim t^{-7.3}$. The predicted powers are $-3,-5$ and -7 , respectively.

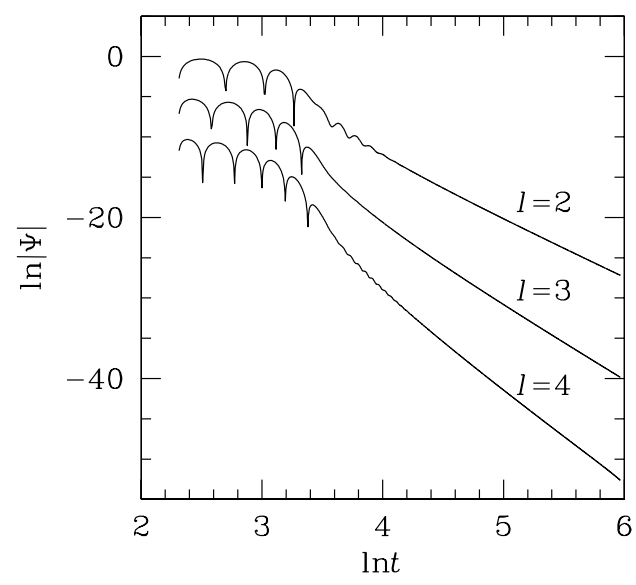

FIG. 2: Generic time dependence of a gravitational Gaussian wavepacket $\Psi$ in a five dimensional Schwarzschild geometry, at a fixed spatial position. The results for the late-time behavior are independent of the initial data. For $l=2$ the late-time behavior is a power-law with $\Psi \sim t^{-7.1}$, for $l=3$ the falloff is given by $\Psi \sim t^{-9.2}$ at late times, and for $l=4$ it is $\Psi \sim t^{-11.4}$. The predicted powers are $-7,-9$ and -11 , respectively. 


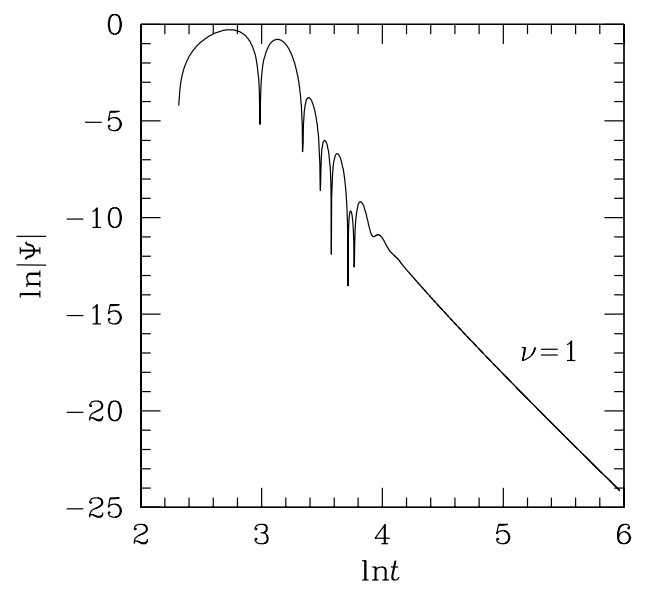

FIG. 3: Evolution of a field subjected to a five-dimensional model potential. The potential is given by (9) but here we take $\nu$ as an integer, $\nu=1$. We obtain at late times $\Psi \sim t^{-6.1}$, whereas the analytical prediction is $t^{-6}$. This shows nicely that for integer $\nu$ the centrifugal barrier contribution vanishes, and it is the next term in the asymptotic expansion of the potential that gives the most important contribution. We have checked that this power law is indeed the correct one, and not a numerical artifact of the ghost potential.

second order scheme. Technically the presence of ghost potentials can be detected by changing the grid size [7]. If the results with different grid sizes are different, then the ghost potential is present. Our numerical results for $D=5$, presented in Figs. 1-3 are free from any ghosts.

The numerical results are in excellent agreement with the analytical predictions (14), and seems moreover to be quite independent of the initial data. This also means that for odd dimensional spacetimes the late time behavior is dictated not by the black hole, but by the fact that spacetime has an odd number of dimensions. To further check that it is in fact the centrifugal barrier term that is controlling the tail, we have performed numerical evolutions with a five dimensional model potential. To be concrete, we have evolved a field subjected to the potential (9) (with $D=5$ ) but we have considered an integer value for $\nu$, namely $\nu=1$. The result is shown in Fig. 3 . Of course the true potential has a semi-integer value for $\nu$, but this way one can verify the dependence of the tail on the centrifugal term. Indeed if $\nu=1$ then by (4), with $\nu=1$ and $\alpha=4$, the late time tail should be $\Psi \sim t^{-6}$. The agreement with the numerical evolution is great. It is therefore the centrifugal barrier that controls the tail in odd dimensional spacetimes. For even dimensional spacetimes, $\nu=l-2+\frac{D}{2}$ is an integer. Moreover, $\alpha=D-1<2 \nu+3=2 l+D-1$. Therefore we are in situation (3). So the late time tail of wave propagation in an even $D$-dimensional Schwarzschild spacetime is a power-law,

$$
\Psi \sim t^{-(2 l+3 D-8)}, \text { even } D
$$

\section{CONCLUSIONS}

We have determined the late time behavior of massless fields (scalar, electromagnetic and gravitational) outside a $D$-dimensional Schwarzschild black hole. For odd $D$, the field at late times has a power-law falloff, $\Psi \sim t^{-(2 l+D-2)}$, and this tail is independent of the presence of the black hole. It depends solely on the flat spacetime background, through the properties of the flat space odd dimensional Green's function. For even $D$, the late time behavior is again a power-law but this time it is due to the presence of the black hole, and is given by $\Psi \sim t^{-(2 l+3 D-8)}$, at late times for $D>4$. We have focused on large extra dimensions only. Recent investigations [17], focusing on brane world models, and therefore compact extra dimensions suggest that the tail is more slowly damped if the extra dimensions are compact. In fact, for $D=5$ they obtain a power-law $\Psi \sim t^{-5 / 2}$ whereas we have $\Psi \sim t^{-3}$ for spherically symmetric perturbations. This may be due to the reflection of the field at the boundaries of the extra dimension.

\section{Acknowledgements}

This work was partially funded by Fundação para a Ciência e Tecnologia (FCT) - Portugal through project PESO/PRO/2000/4014. SY acknowledges finantial support from FCT through project SAPIENS 36280/99. VC and OD also acknowledge finantial support from FCT through PRAXIS XXI programme. JPSL acknowledges finantial support from ICCTI/FCT and thanks Observatório Nacional do Rio de Janeiro for hospitality.
[1] B. S. DeWitt and R. W. Brehme, Ann. Phys. (NY)9 220 (1965).

[2] R. H. Price, Phys. Rev. D5, 2419 (1972).

[3] C. Gundlach, R. H. Price and J. Pullin, Phys. Rev. D49, 883 (1994).

[4] C. Gundlach, R. H. Price and J. Pullin, Phys. Rev. D49, 890 (1994).

[5] E. Leaver, Phys. Rev. D34, 384 (1986).
[6] E. S. C. Ching, P. T. Leung, W. M. Suen and K. Young, Phys. Rev. Lett. 74, 2414 (1995).

[7] E. S. C. Ching, P. T. Leung, W. M. Suen and K. Young, Phys. Rev. D52, 2118 (1995).

[8] H. Koyama and A. Tomimatsu, Phys. Rev. D64, 044014 (2001).

[9] S. Hod, Class. Quant. Grav. 18, 1311 (2001).

[10] J. Bekenstein, in Cosmology and Gravitation, edited by 
M. Novello (Atlasciences, France 2000), pp. 1-85, grqc/9808028 (1998).

[11] For a review on quasinormal modes and their astrophysical importance in asymptotically flat spacetimes see: K. D. Kokkotas and B. G. Schmidt, Living Rev. Rel. 2, 2(1999); H. -P. Nollert, Class. Quant. Grav. 16, R159 (1999).

[12] For recent developments relating quasinormal modes with decay timescales in the AdS/CFT see G. T. Horowitz and V. E. Hubeny Phys. Rev. D62, 024027 (2000); V. Cardoso and J. P. S. Lemos, Phys. Rev. D64, 084017 (2001); Phys. Rev. D63, 124015 (2001); R. A. Konoplya, Phys. Rev. D66, 044009 (2002); E. Berti and K.D. Kokkotas, Phys. Rev. D67, 064020 (2003). Very recently it has been proposed that there exists a relation between highly damped quasinormal modes and Loop Quantum Gravity. See for example: S. Hod, Phys. Rev. Lett. 81, 4293 (1998); O. Dreyer, Phys. Rev. Lett. 90, 081301 (2003); L. Motl, A. Neitzke, hep-th/0301173 (2003); A. Maassen van den Brink, gr-qc/0303095 (2003); D. Birmingham, S. Carlip, Y. Chen, hep-th/0305113 (2003); E. Berti, K. D. Kokkotas, hep-th/0303029 (2003); V. Cardoso, J. P. S. Lemos, Phys. Rev. D67, 084020 (2003); V. Cardoso, R. Konoplya, J. P. S. Lemos, grqc/0305037 (2003); E. Berti, V. Cardoso, K. D. Kokko- tas, H. Onozawa, hep-th/0307013; N. Andersson, C.J. Howls, gr-qc/0307020.

[13] S. Hassani, Mathematical Physics, (Springer-Verlag, New York, 1998); R. Courant and D. Hilbert, Methods of Mathematical Physics, Chapter VI (Interscience, New York, 1962).

[14] V. Cardoso, O. J. C. Dias and J. P. S. Lemos, Phys. Rev. D67, 064026 (2003).

[15] H. Soodak and M. S. Tiersten, Am. J. Phys. 61, 395 (1993).

[16] N. Arkani-Hamed, S. Dimopoulos and G. Dvali, Phys. Lett. B429, 263 (1998); Phys. Rev. D59, 086004 (1999); I. Antoniadis, N. Arkani-Hamed, S. Dimopoulos and G. Dvali, Phys. Lett. B436, 257 (1998).

[17] A. O. Barvinsky, S. N. Solodukhin, hep-th/0307011.

[18] F. R. Tangherlini, Nuovo Cim. 27, 636 (1963); R. C. Myers and M. J. Perry, Annals Phys. 172, 304 (1986).

[19] V. Cardoso, J. P. S. Lemos, Phys. Rev. D66, 064006 (2002).

[20] H. Kodama, A. Ishibashi, hep-th/0305147 (2003).

[21] T. Regge, J. A. Wheeler, Phys. Rev. 108, 1063 (1957).

[22] F. Zerilli, Phys. Rev. Lett. 24, 737 (1970).

[23] L. C. B. Crispino, A. Higuchi, G. E. A. Matsas, Phys. Rev. D63, 124008 (2001). 